\title{
Note on "An efficient approach for solving the lot-sizing problem with time-varying storage capacities"
}

\author{
Wilco van den Heuvel*a, José Miguel Gutiérrez ${ }^{\mathrm{b}}$, And \\ HARK-CHIN HWANG ${ }^{c}$
}

${ }^{a}$ Econometric Institute and Erasmus Research Institute of Management, Erasmus University Rotterdam, P.O. Box 1738, 3000 DR Rotterdam, The

Netherlands, wvandenheuvel@ese.eur.nl

${ }^{\text {b} D p t o . ~ d e ~ E s t a d i ́ s t i c a, ~ I . O . ~ y ~ C o m p u t a c i o ́ n, ~ U n i v e r s i d a d ~ d e ~ L a ~ L a g u n a, ~}$ Tenerife, Islas Canarias, Spain, jmgrrez@ull.es

${ }^{\mathrm{c}}$ Department of Industrial Engineering, Chosun University 375 Seosuk-Dong, Dong-Gu, Gwangju 501-759, South Korea, hchwang@chosun.ac.kr

ECONOMETRIC Institute Report EI 2010-70

\begin{abstract}
In a recent paper Gutiérrez et al. (2008) show that the lot-sizing problem with inventory bounds can be solved in $\mathcal{O}(T \log T)$ time. In this note we show that their algorithm does not lead to an optimal solution in general.
\end{abstract}

*Corresponding author. Tel.: +31 10 4081307; fax: +31 104089162 
Keywords: Inventory, Lot-sizing, Inventory bounds

\section{Introduction}

The lot-sizing problem with inventory bounds (LSB) is described as follows. Given the (deterministic) demands for a finite planning horizon of length $T$, find a production plan at minimal cost such that all demand is satisfied, while the inventory level in each period should be no larger than the storage capacity. Toczylowski (1995) solved this problem in $\mathcal{O}\left(T^{2}\right)$ time. Recently, Gutiérrez et al. (2008) improved the running time to $\mathcal{O}(T \log T)$ time. In this note, we show that the algorithm of Gutiérrez et al. (2008) does not lead to an optimal solution in general.

This note is organized as follows. In Section 2 we formulate the problem. In Section 3 we briefly describe the geometric technique of Wagelmans et al. (1992), which is applied in Gutiérrez et al. (2008). Furthermore, in Section 4 we show why the algorithm of Gutiérrez et al. (2008) does not lead to an optimal solution in general. Finally, this note ends with some concluding remarks in Section 5.

\section{Problem description}

To describe the LSB, we use the same notation as Gutiérrez et al. (2008). For $t=1, \ldots, T$ we let

$d_{t}: \quad$ demand in period $t$ with $d_{i, j}=\sum_{t=i}^{j} d_{t}$

$f_{t}: \quad$ setup cost in period $t$

$p_{t}$ : unit production cost in period $t$

$h_{t}$ : unit holding cost in period $t$

$S_{t}$ : inventory bound in period $t$

$y_{t}$ : binary setup variable in period $t$

$x_{t}$ : production quantity in period $t$

$I_{t}$ : ending inventory in period $t$. 
Given this notation, the problem is formulated as

$$
\begin{array}{lll}
\min & \sum_{t=1}^{T}\left(f_{t} y_{t}+p_{t} x_{t}+h_{t} I_{t}\right) & \\
\text { s.t. } & I_{t}=I_{t-1}+x_{t}-d_{t} & \text { for } t=1, \ldots, T \\
& x_{t} \leq d_{t, T} y_{t} & \text { for } t=1, \ldots, T \\
& I_{t-1}+x_{t} \leq S_{t} & \text { for } t=1, \ldots, T \\
& I_{t}, x_{t} \geq 0, y_{t} \in\{0,1\} & \text { for } t=1, \ldots, T,
\end{array}
$$

where we assume that $I_{0}=I_{T}=0$. Note that the bound $S_{t}$ is imposed on the starting inventory $I_{t-1}+x_{t}$ in period $t$. Using the inventory balance constraint, this constraint can also be written as $I_{t} \leq S_{t}-d_{t}$. For feasibility we need that $S_{t} \geq d_{t}$ for $t=1, \ldots, T$.

\section{Geometric technique of Wagelmans et al. (1992)}

Because Gutiérrez et al. (2008) apply the geometric technique of Wagelmans et al. (1992), we briefly describe this technique. First, let $F(t)$ be the optimal cost for periods $t, \ldots, T$ in case of no inventory bounds. Using the zero-inventory property (Wagner and Whitin, 1958), the problem can be solved by the recursion

$$
\begin{aligned}
F(t) & =\min _{t<j \leq T+1}\left\{f_{t}+c_{t} d_{t, j-1}+F(j)\right\} \\
& =f_{t}+\min _{t<j \leq T+1}\left\{F(j)+c_{t} d_{t, j-1}\right\}
\end{aligned}
$$

where we made the common substitution $c_{t}=p_{t}+\sum_{i=t}^{T} h_{i}$ and we let $F(T+1)=0$. Assume that the values $F(t)$ are known for $t=j+1, \ldots, T+1$. Then we can plot the points $\left(d_{t, T}, F(t)\right)$ for $t=j+1, \ldots, T+1$ and determine the lower envelope of this set of points (see Figure 1). The points that contribute to the lower envelope are called efficient periods, while the non-contributing points are called non-efficient.

Given the lower envelope of these points, Wagelmans et al. (1992) show that the minimum in (1), and hence the value of $F(j)$, can be found in $\mathcal{O}(\log T)$ time by finding the point that is tangent to the line with slope $c_{j}$ (see Figure 1). Furthermore, they show that 
adding the new point $\left(d_{j, T}, F(j)\right)$ and updating the lower envelope takes $\mathcal{O}(T)$ time in the total execution of the algorithm. This means that the overall running time is $\mathcal{O}(T \log T)$.

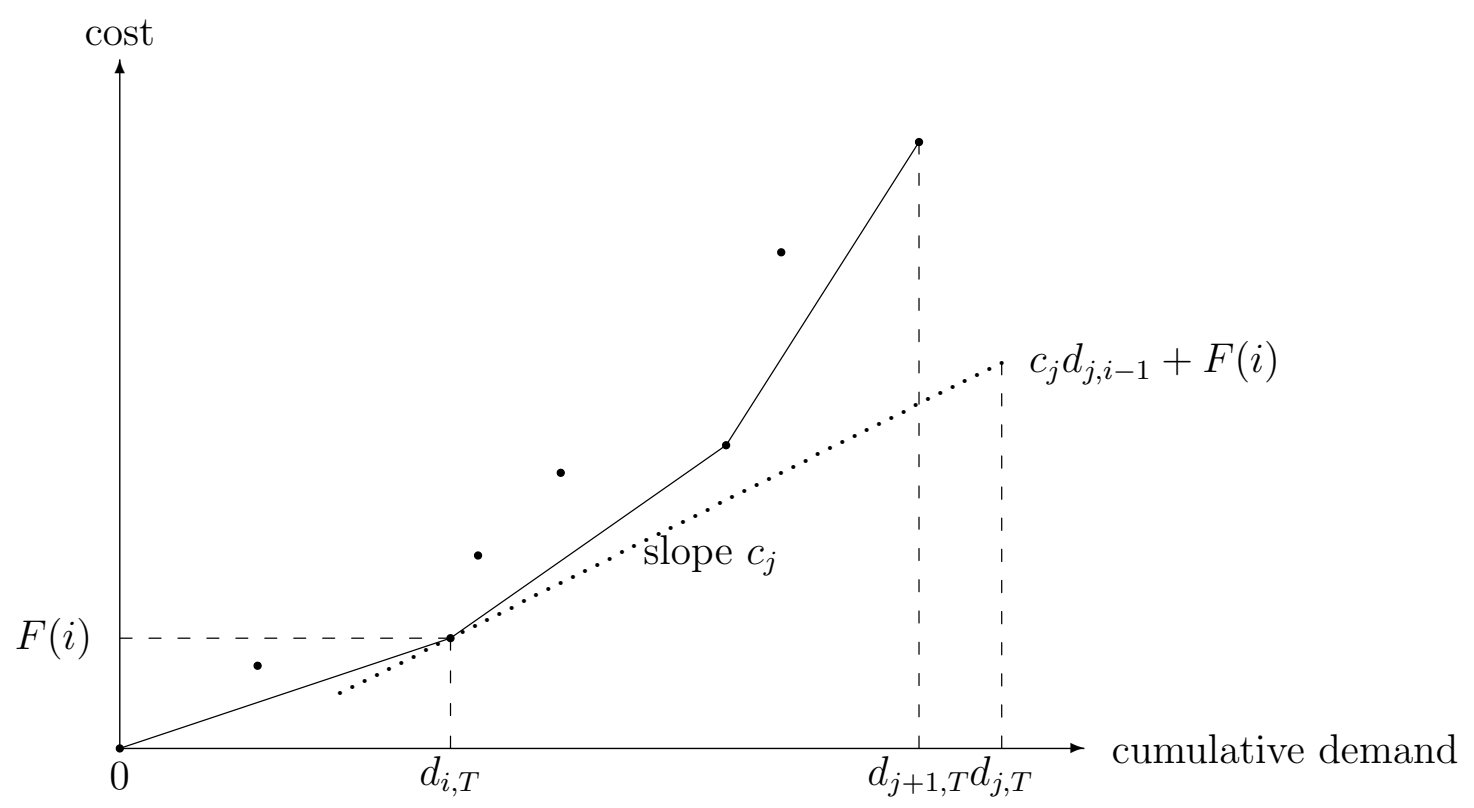

Figure 1: Illustration of the geometric technique of Wagelmans et al. (1992)

\section{Mistakes in Gutiérrez et al. (2008)}

Gutiérrez et al. (2008) apply the technique of Wagelmans et al. (1992) to solve the problem with inventory bounds. To that end, they use the recursive variable $G(t)$, the optimal cost to satisfy the demands in periods $t, \ldots, T$. Before giving the recursion formula, we need some more notation. Let $\hat{x}_{t}$ be the optimal production quantity in period $t$ corresponding to $G(t)$. Furthermore, let $M_{t}$ be the maximum production quantity in period $t$, i.e., $M_{t}=\min \left\{d_{t, j-1}+S_{j}: j=t, \ldots, T\right\}$. Finally, let $R_{t}$ be the largest period that can be completely satisfied by production in period $t$, i.e., $R_{t}=\max \left\{t \leq j \leq T: d_{t, j} \leq M_{t}\right\}$.

If we consider the problem starting from period $t$, then Love (1973) and Gutiérrez et al. (2008) show that one of the following properties holds in an optimal solution: (i) the production quantity is equal to the sum of an integer number of consecutive demands 
starting at period $t$, or (ii) the production quantity is equal to the maximum production quantity $M_{t}$. Given these properties the LSB can be solved by the recursion (for ease of exposition we assume that $d_{t}>0$ )

$$
\begin{aligned}
G(t) & =\min \left\{\begin{array}{l}
\min _{t<j \leq R_{t}+1}\left\{f_{t}+c_{t} d_{t, j-1}+G(j)\right\} \\
\min _{\left\{t<j \leq R_{t}+1: \hat{x}_{j} \geq M_{t}-d_{t, j-1}\right\}}\left\{f_{t}+c_{t} M_{t}-c_{j}\left(M_{t}-d_{t, j-1}\right)+G(j)\right\}
\end{array}\right. \\
& =f_{t}+\min \left\{\begin{array}{l}
\min _{t<j \leq R_{t}+1}\left\{c_{t} d_{t, j-1}+G(j)\right\} \\
\min _{\left\{t<j \leq R_{t}+1: \hat{x}_{j} \geq M_{t}-d_{t, j-1}\right\}}\left\{c_{t} d_{t, j-1}+G(j)+\left(c_{t}-c_{j}\right)\left(M_{t}-d_{t, j-1}\right)\right\}
\end{array}\right.
\end{aligned}
$$

Note that the term $\left(c_{t}-c_{j}\right)\left(M_{t}-d_{t, j-1}\right)$ corresponds to the cost of producing an amount of $M_{t}-d_{t, j-1}$ in period $t$ instead of in period $j$. This amount should be lower than the production quantity $\hat{x}_{j}$ and so the condition $\hat{x}_{j} \geq M_{t}-d_{t, j-1}$ is needed for feasibility.

Gutiérrez et al. (2008) now proceed as follows. To calculate $G(j)$, they utilize the lower envelope of the points $\left(d_{t, T+1}, G(t)\right)$ for $t=j+1, \ldots, T+1$. Let $q(j)$ be the period corresponding to the point tangent to the line with slope $c_{j}$. Note that the $q(j)$ is equal to period $i$ in Figure 1 and equal to period $r$ in Figure 2. Gutiérrez et al. (2008) use this period to identify the minima in (2).

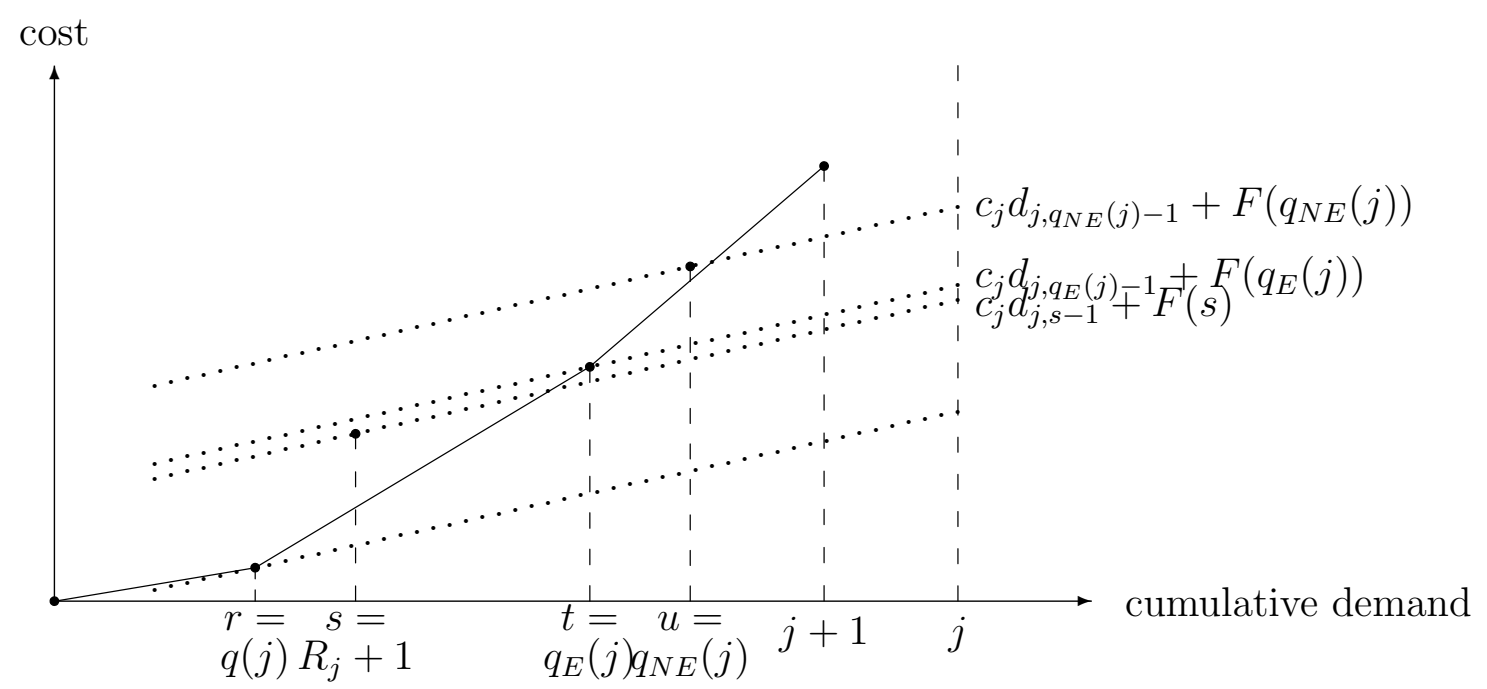

Figure 2: Minimum attained at a non-efficient period (for ease of notation, an index $k$ at the horizontal axis represents the cumulative demand $d_{k, T}$ ) 
We will now show that the algorithm does not necessarily find these minima in case $q(j)>R_{j}+1$. Note that in this case it is not feasible to produce $d_{j, q(j)-1}$ units, because of the inventory bounds. Therefore, Gutiérrez et al. (2008) determine the largest efficient and non-efficient period smaller or equal than $R_{j}+1$ with the smallest slope ratios, denoted by $q_{E}(j)$ and $q_{N E}(j)$, respectively. The slope ratio of some period $t$ is the slope of the line between the point corresponding to period $t$ and the point corresponding to the successor of period $t$ in the lower envelope. They claim that the value $G(j)$ is found (i) by producing for a consecutive number of periods up to period $q_{E}(j)-1$ or up to $q_{N E}(j)-1$, or (ii) to produce $M_{j}$ units in period $j$ and to have the next production in period $q_{E}(j)$ or $q_{N E}(j)$.

The mistake in case (i) is that the period that minimizes the first term in (2) is not necessarily equal to period $q_{E}(j)$ or $q_{N E}(j)$. This is graphically illustrated in Figure 2. It follows from this figure that the optimal period is $s$, which is neither equal to $q_{E}(j)$ nor to $q_{N E}(j)$. Note that $q_{N E}(j)=u$ because the slope ratio of period $u\left((G(u)-G(t)) / d_{u, t-1}\right)$ is smaller than the slope ratio of period $s\left((G(s)-G(r)) / d_{s, r-1}\right)$. To overcome this mistake one should use the lower envelope of the points $\left(d_{t, T+1}, G(t)\right)$ for $t=j+1, \ldots, R_{j}+1$. However, this means that in every iteration the left part of the lower envelope needs to be updated, which takes additional computation time. An issue for case (ii) is that Gutiérrez et al. (2008) do not check in their algorithm whether the condition $\hat{x}_{j} \geq M_{j}-d_{j, i-1}$ holds for $i \in\left\{q_{E}(j), q_{N E}(j)\right\}$. Hence, the periods $q_{E}(j)$ and $q_{N E}(j)$ may correspond to a nonfeasible solution. Furthermore, this means that the (feasible) periods $q_{E}(j)$ and $q_{N E}(j)$ can not be easily found by binary search.

A more fundamental mistake is that Gutiérrez et al. (2008) try to find the minimum of the second term in (2) by utilizing the lower envelope of the points $\left(d_{t, T}, G(t)\right)$ for $t=j+1, \ldots, T$. However, the term $\left(c_{t}-c_{j}\right)\left(M_{t}-d_{t, j-1}\right)$ causes that the approach of Wagelmans et al. (1992) cannot be applied anymore. If this term is added to every value $G(j)$ at the start of an iteration, then the approach still works. However, this means that each point and the corresponding lower envelope should be recalculated in every iteration, which implies that the order of $\mathcal{O}(T \log T)$ running time cannot be achieved anymore. The following numerical example shows that the periods $q(j)$ and $q_{E}(j)$ cannot be used to 
identify the minimum of the second term in (2), implying that the algorithm of Gutiérrez et al. (2008) fails to find an optimal solution in general.

Example 1 Consider the 3-period problem instance of Table 1. It follows that $R_{1}=2$ and $R_{2}=R_{3}=3$, which means that the problem starting from period 2 is uncapacitated. After execution of the algorithm (Gutiérrez et al., 2008, p. 690, Algorithm 1), we get the following values (we only present the most relevant values), where $L E$ denotes the set of efficient periods in the lower envelope:

Initialization: $\quad G(4)=0, L E=\{4\}$ (we assume that $c_{4}=0$ although this is not specified in the algorithm)

Iteration 1: $\quad q(3)=4, G(3)=4, L E=\{4,3\}$

Iteration 2: $\quad q(2)=3, G(2)=8.2, L E=\{4,3,2\}$

Iteration 3: $\quad q(1)=4>3=R_{1}+1, q_{E}(1)=3$ and $c_{1}<c_{3}, G(1)=3$ (obtained from code line 19 in Algorithm 1)

So the solution obtained from the algorithm is: $x_{1}=5$ and $x_{3}=1$ with cost 3 . However, the optimal solution is: $x_{1}=5$ and $x_{2}=1$ with cost 2.1. Therefore, the optimal solution is not found by Algorithm 1 of Gutiérrez et al. (2008).

\begin{tabular}{c|ccc}
\hline$t$ & 1 & 2 & 3 \\
\hline$d_{t}$ & 2 & 2 & 2 \\
$f_{t}$ & 0 & 0 & 2 \\
$c_{t}$ & 0 & 2.1 & 1 \\
$M_{t}$ & 5 & 4 & 2 \\
\hline
\end{tabular}

Table 1: Problem instance corresponding to Example 1 


\section{Concluding remarks}

The question remains whether the lot-sizing problem with inventory bounds can be solved in $\mathcal{O}(T \log T)$ time. As follows from this note, to find the minimum in the first term of (2), one needs to find an algorithm that updates the left part of the lower envelope in $\mathcal{O}(T \log T)$ time. Furthermore, it seems that one needs another recursion to find the minimum in the second term of (2), as the lower envelope does not provide the required information. It is shown in Hwang and van den Heuvel (2010 (under revision) how these issues can be resolved for a more general problem. They develop an $\mathcal{O}(T \log T)$ time algorithm for the lot-sizing problem with inventory bounds and back-logging.

\section{References}

J. Gutiérrez, A. Sedeño-Noda, M. Colebrook, and J. Sicilia. An efficient approach for solving the lot-sizing problem with time-varying storage capacities. European Journal of Operational Research, 189:682-693, 2008.

H.-C. Hwang and W. van den Heuvel. Improved algorithms for a lot-sizing problem with inventory bounds and backlogging. Technical Report EI 2010-17, Econometric Institute, Erasmus University Rotterdam, 2010 (under revision).

F. Love. Bounded production and inventory models with piecewise concave costs. Management Science, 20:313-318, 1973.

E. Toczylowski. An $O\left(T^{2}\right)$ algorithm for the lot-sizing problem with limited inventory levels. IEEE Symposium on Emerging Technologies 86 Factory Automation, 3:78-85, 1995.

A. P. M. Wagelmans, C. P. M. van Hoesel, and A. Kolen. Economic lot sizing: An $\mathrm{O}(n \log n)$ algorithm that runs in linear time in the Wagner-Whitin case. Operations Research, 40: S145-S156, 1992. 
H. M. Wagner and T. M. Whitin. Dynamic version of the economic lot size model. Management Science, 5:89-96, 1958. 